Final manuscript for Springer book 'Architecture and Interaction. Human Computer Interaction in Space and Place' edited by Dalton, Schnädelbach, Wiberg and Varoudis in series 'Human-Computer Interaction', Forthcoming in 2016

\title{
Measuring Interaction in Workplaces
}

\author{
Dr Kerstin Sailer ${ }^{1}$, Petros Koutsolampros ${ }^{1}$, Dr Martin Zaltz Austwick ${ }^{2}$, Dr \\ Tasos Varoudis ${ }^{1}$, Dr Andy Hudson-Smith ${ }^{2}$
}

\author{
1 Space Syntax Laboratory, The Bartlett School of Architecture, University College London, \\ 140 Hampstead Road, London NW1 2BX; \\ 2 Centre for Advanced Spatial Analysis (CASA), The Bartlett, University College London, \\ 90 Tottenham Court Road, London, W1T 4TJ \\ Corresponding author: Dr Kerstin Sailer: k.sailer@ucl.ac.uk
}

\begin{abstract}
Interactions in the workplace have long been studied by the architectural research community, however, in the past, the majority of those contributions focused on single case studies. Drawing on a much larger empirical sample of 27 offices, this chapter aims at establishing a baseline of understanding how the physical structure of office buildings shapes human behaviours of interaction. This may form a foundation for the Human-Computer Interaction (HCI) community to investigate the impact of embedded computer technology on human behaviours inside buildings. Methods of data collection included an analysis of floor plans with Space Syntax techniques and direct observations of space usage patterns. Exploring this data, different patterns emerged: interactions appeared unevenly distributed in space; interaction rates as well as preferences for locations varied by industry; spatial configuration appeared to create affordances for interaction, since unplanned interactions outside of meeting rooms tended to cluster in more visually connected areas of the office; in addition, seven different micro-behaviours of interaction were identified, each of them driven by affordances in both the built environment and the presence of other people; last but not least, locations for interactions showed clear time-space routines. The chapter closes with interpretations of the results, reflecting on the problem of predictability and how these insights could be useful for evidencebased design, but also the HCI community. It also gives an outlook on future developments regarding the constant logging of human behaviours in offices with emerging technologies.
\end{abstract}

\section{Keywords}

Interaction; Face-to-Face; Workspaces; Offices; Space Syntax; Visualisation; 


\title{
Introduction - Interaction, Space and Architectural Typologies
}

\author{
"Culturally and socially, space is never simply the inert background of our material \\ existence. It is a key aspect of how societies and cultures are constituted in the real world, \\ and, through this constitution, structured for us as 'objective' realities. Space is more than \\ a neutral framework for social and cultural forms. It is built into those very forms. Human \\ behaviour does not simply happen in space. It has its own spatial forms. Encountering, \\ congregating, avoiding, interacting, dwelling, teaching, eating, conferring are not just \\ activities that happen in space. In themselves they constitute spatial patterns." (Hillier \\ 1996, p.29)
}

Interaction is one of the key aspects of human life. According to Hillier interaction is embedded in social and cultural forms, and alongside many other activities, it forms spatial patterns as an integral part of everyday life (Goffman 1959).

Human-to-human interaction is driven by the spatial setting in which it occurs. Interaction in digital space, for instance, offers more opportunities for anonymity than interaction in physical space. Likewise, interaction in urban space offers more opportunities for anonymity than interaction inside buildings, where one can expect to meet more like-minded people or even familiar faces. Inside buildings, interactions are shaped by the type of building (hospital, school, office, museum, department story, library, etc.), but also by the properties of the layout. Indeed, it has been argued that buildings have two main functions in this respect (Hillier and Hanson 1984, Hillier et al. 1984): firstly, they define users by categories: as soon as we enter for instance a hospital, we assume a particular role as patient, visitor, nurse, doctor, administrator, cleaner, porter etc. Buildings then transform our experience of space by granting or inhibiting access and control, not only over space, but also with regards to the production and reproduction of social knowledge inscribed in the building. It turns us into visitors with limited access and control, or inhabitants who define the building and its outputs. Secondly, buildings by virtue of their spatial layout provide mechanisms for patterns of avoidance or encounter between the relevant user groups. This aspect of building function has been coined 'the construction of an interface' (Hillier et al. 1984).

Workplaces are interesting cases of buildings to investigate in this interdisciplinary context of Architecture and Interaction for a variety of reasons: firstly, offices are a building typology characterized by a rather unstructured interface and subtle role assignments. There are no sharp contrasts between user groups (such as exist in a hospital), and office buildings do not programmatically limit contact, hence the detailed spatial configuration and strategic usage choices (for instance where to put the coffee machine and other attractors) plays an important role in shaping user behaviours and contact patterns (Sailer 2007). Secondly, they afford the potential for observational studies and field-testing of real world applications (Shklovski and Chang 2006) crucial to the field of Human Computer Interaction (HCI). They also have the capacity to provide a large number of participants for data collection purposes whilst ensuring high ecological validity of the results - the extent to which behaviour observed in an experiment reflects behaviour that occurs naturally. Last but not least, technology has entered the scene 
and has over the last decades if not centuries clearly changed the way in which work is structured. This renders them interesting for the wider debate initiated by this book. The bigger picture of the changing landscape of work is relevant, since predictions of the 'death of distance' (Cairncross 1997) have resonated in a lively debate on whether technology has made office buildings obsolete, allowing work to leave the building and (in theory) taking place anywhere anytime, afforded by increased mobility of computing devices such as laptops, tablets or smartphones and the rise in internet connectivity on the go (Rainie and Wellman 2012). While it is unquestionable that technology has changed the way people work, it is equally clear that office buildings are as much needed as ever, evident in the recent renaissance in prestigious architecture projects of large and prominent office buildings, ironically many of them for big technology corporations, such as Facebook (Gehry), Apple (Foster's \& Partner), Samsung (NBBJ) and Google (BIG and Heatherwick Studio), to name just a few. Likewise, Yahoo has been in the news in 2013, asking its employees to quit home working and instead return to the office to enable "hallway and cafeteria discussions, meeting new people, and impromptu team meetings (...) [where] some of the best decisions and insights come from" Scholarly research has shown repeatedly that face-to-face interactions allow for rich information exchanges, in particular rapid feedback, high frequency, instantaneous feedback, learning, visual and body language cues, trust, relationship building, socialising and motivation (Nohria and Eccles 1992, Storper and Venables 2004). It was also shown that face-to-face interaction patterns often match digitallymediated interaction patterns in offices very closely (Sailer et al. 2015), hence the opportunity to overcome physical space offered by technology is not always realized. This embeds our study in the tradition of considering 'situated architectural effects' and the role of technology in everyday life (Fischer and Hornecker 2012).

Against this background, this chapter seeks to explore patterns of human-tohuman face-to-face interactions in the workplace mediated by the structure of the physical office layout. Its main research question is grounded in the understanding that interaction constitutes a spatial pattern and aims to discover how interactions are distributed in workplaces, which spatial settings provide favourable conditions for interactions to flourish and to which degree different offices show varying or converging phenomena. This is relevant to the HCI community insofar as it establishes a baseline of understanding of how physical space structures human behaviours in offices. Marshall et al. (2011) proposed that there is a lack of understanding the affordances of space prior to an HCI intervention. Hence, on the foundation of this chapter, future research could test how human behaviour shifts with the impact of embedded computer technology, such as screens and displays in line with HCI relevant research questions.

The chapter is structured as follows: section 2 will provide a brief background of relevant literature and continue contextualizing the understanding of human interactions in physical space in the wider discourse on HCI. Section 3 will

\footnotetext{
${ }^{1}$ The leaked internal Yahoo memo is documented here: http://allthingsd.com/20130222/physically-together-heres-the-internal-yahoo-no-work-fromhome-memo-which-extends-beyond-remote-workers/ (Last accessed: 01 April 2015)
} 
introduce methodology and the detailed empirical dataset used in this research. Results on locational patterns and the spatial distribution of interaction occurrences in workplaces will be discussed in section 4, giving rise to an interpretation of predominant spatial cultures and practices of interaction in offices. Highlighting future work and overall developments in the field, section 5 will provide an outlook on where technology might take the world of work and offices, thus building more bridges to bring architectural research and advanced spatial analysis together with the HCI community.

\section{Architecture and Human Interaction}

In this context, three relevant bodies of literature are relevant and will be sketched in the following: firstly, scholarly work tackling the question of how to capture interactions in offices; secondly, contributions that highlight the impact of spatial layout on patterns of interaction in workplaces; and thirdly, literature bringing HCI to bear in the context of workplaces and human interaction patterns.

The question of how to capture and measure interaction in the office accurately is an interesting one in the context of this chapter. Traditionally, researchers have relied on methods stemming from the social sciences such as direct observations or spot sampling (Reiss 1971, Bernard 2000), or staff surveys of interaction patterns (Bernard 2000). In recent years, automated methods of collecting interaction data in the workplace have been deployed using sensing systems (Wu et al. 2008, Lopez de Vallejo 2009, Olguin et al. 2009, Brown et al. 2014a, Brown et al. 2014b). Traditional methods of observations and surveys may suffer from problems including validity (Bernard and Killworth 1993), classification errors (in recording ambiguous behaviours such as passive listening), interpretation bias (by respondents completing surveys) (Bernard 2000) or response and recall bias in surveys (Bradburn et al. 1978, Van de Mortel 2008). Still it seems that sensor derived data is far from being as 'objective' as it is often claimed. Interactions are often based on probabilities since not every behavioural instance can be recorded (for instance Olguin et al. 2009 reported a likelihood of $87 \%$ of a face-to-face interaction being captured by their system). Additional issues include accuracy and spatial resolution of the data (the best systems achieve a spatial resolution of the order of a few metres, which can still be insufficient for understanding microbehaviours within organisations), scalability and affordability, ethical problems as well as calibration issues and context-dependency (certain building materials such as glass and steel may cause interference and reflection). Comparing traditional ways of gathering interaction data in the workplace with sensor derived data typically showed a low correlation (Sailer et al. 2013), highlighting the fact that technical systems cannot yet replace sociological human enquiry. Despite remaining issues with indoor location and interaction sensing (Curran et al. 2011, $\mathrm{Li}$ and Becerik-Gerber 2011), this growing field may provide future opportunities for architectural and HCI research to come together. 
The second relevant stream of literature regards the impact of workplace layout on social behaviours in general, and patterns of interaction in particular. This has been studied by various scholars over the last decades, with a majority of the contributions coming from the tradition of Space Syntax, which is a method and theory aiming to understand the relationship between spatial configuration (the way spatial elements are interconnected and form part of a wider spatial network), and social behaviours. Research in this domain has found that workplaces with an overall shorter path length in the spatial network (more integrated buildings in Space Syntax terminology) showed a higher degree of interaction among staff (Hillier and Grajewski 1990, Toker and Gray 2008) and a higher connectivity between teams (Hillier and Grajewski 1990). Interactions among staff were found to take place predominantly around desks and workstations (Steen et al. 2005, Rashid et al. 2006, Markhede and Koch 2007, Steen 2009); attractors such as watercoolers and photocopiers invited informal interactions especially in integrated locations, but only if they matched an organisation's culture and behavioural norms and respected the need for privacy of conversations (Fayard and Weeks 2007). The importance of paths in the workplace was underlined by showing how interactions often arose out of temporary proximity between people, where one person was on the move and was 'recruited' into a conversation by someone sitting (Backhouse and Drew 1992). In addition, more permanent patterns of proximity between coworkers as measured by the walking distance between their desks was associated with frequency of interaction (Sailer and Penn 2009) but also the structure of interaction networks (Sailer and McCulloh 2012).

Last but not least, research in the HCI field has investigated human interaction patterns in workplace environments. $\mathrm{HCI}$ is a young discipline which has developed over the last thirty years. The origins of the discipline are within computer science and cognitive psychology, with influences from sociology, anthropology, ergonomics and design. The multidisciplinary nature of $\mathrm{HCI}$ means there are numerous methodological approaches employed to conduct research in the area and ways in which $\mathrm{HCI}$ research connects to architectural research relevant to the study at hand. A commonly used framework in HCI research that bridges to architectural research is Hall's proxemics approach (Hall 1966), highlighting how the nature of interactions between people changes with the distance between them. This has been used in HCI to understand both human-to-human, but also human-computer interaction, for instance in an analysis of media facades (Fischer and Hornecker 2012). A particular example of relevant HCI research inside workplaces is the so called mixed reality architecture (MRA) system, which connects office occupants to selected collaborators or colleagues in other locations. Cameras display one location A to a large screen in location B with microphones / speakers allowing an additional audio-connection (if the occupants have chosen to connect, similarly to opening their door in an office). Research in this area proposed that awareness of others and social interactions as well as chance encounters were realized between remote locations in similar ways in which a shared physical space supports face-toface interaction (Schnädelbach et al. 2006). However, it was also shown that virtual adjacencies between remote locations via an MRA created distinct spatial topologies of a hybrid nature, where configurations changed in a dynamic and rapid 
way (Schnädelbach 2012). Interestingly, interactions with strangers were not afforded by the system unless people were formally introduced to each other, which highlights the ways in which workplace interactions mediated by screens might differ from face-to-face interactions, where conversations between strangers, for instance at the coffee machine, do happen occasionally. The study of ambient displays in workplaces highlighted that people adapted their micro-behaviour of movement if screens augmented their visual field (Varoudis 2011). It was also shown how ambient displays were able to nudge people's behaviour in workplaces, in this case to increase use of the stairs versus the elevators (Rogers et al. 2010). These approaches pinpoint future research opportunities combining the architecturally motivated research of behaviours with augmentations provided by digital spaces.

In summary, four themes appear relevant for the study of interaction patterns in offices from the perspective of architectural and HCI research: firstly, both research traditions consider the environment as a variable that delivers data on the nature of human interactions. Similarities as well as differences between interactions in physical and augmented reality were observed. Secondly, interactions are framed as part of the user experience, both in physical space (architectural research) and in digital space (HCI). Thirdly, interactions are defined and shaped by the construction of an interface. In architectural research this relates to the way buildings define user groups and systematically facilitate or hinder encounters between the groups by controlling access and visibility. In the case of HCI research, the interface is the connection between human and computer, allowing or hindering user actions and interactions. Fourthly, new research opportunities between architectural and HCI research were seen in understanding how affordances for interaction are structured across physical and digital spaces, but also on a methodological basis in a quest to advance the locational accuracy of sensing systems.

\section{Data and Methods}

This paper draws on a large sample of 27 different cases of offices collected by Spacelab, a design and consultancy practice based in London and organized into a Spatial Database during the Knowledge Transfer Partnership Project 'Big data in the office' between UCL and Spacelab. The database (described in more detail in Koutsolampros et al. 2015) contains information on 27 buildings (however, different offices in different locations belonging to the same company have been treated as standalone cases). In total, data from 14 companies across industries such as Media, Advertising, Legal, Technology, Retail and Financial Services was analysed. The cases varied in size, from 400 to $15000 \mathrm{~m}^{2}$ of office area and 40 to 1700 staff.

In each of the cases, the following methods of data collection and enquiry were combined: firstly, structured participant observations of space usage, so called snapshots (Grajewski 1992) recorded and mapped different behaviours (i.e. people sitting, standing, walking and interaction) to their exact location using a tailor-made 
tablet application. Every area was observed in hourly intervals 8 times per day over the course of a full working week, resulting in a total of 40 observations of each space. This was achieved for 20 buildings. The remaining 7 buildings were observed with fewer snapshots (mostly 3 days).

Secondly, the functional allocation of spaces was mapped onto floor plans, showing where open plan and cellular workspaces, alternative workspaces (e.g. breakout spaces), meeting rooms, primary circulation and shared facilities (e.g. kitchens, canteens, tea points) were located. These functional definitions were developed from expert observation of the floor plans and actual space in the course of Spacelab's contracts ${ }^{2}$. Floor plans were also processed in a Space Syntax analysis. Visibility graph analysis (VGA) (Turner et al. 2001) was conducted on eye-level using depthmapX software (Varoudis 2012), which divides the office space into a regular grid $(0.45 \times 0.45 \mathrm{~m})$ and determines the number of steps required to establish visibility between any pair of grid points (or pixels); two grid points are defined as one step of depth apart when they are visible to one another. This metric of average mean depth (MD) of visibility path length was used.

Last but not least, visualisations were created that combine two metrics of interest: in the following figures we show the comparison of visual mean depth (an attribute of space) with the observed interaction density (an attribute of the people using the space, and their social and cultural structures). To make the two metrics comparable, interaction was converted from a list of discrete locations where interaction has occurred to a continuous field indicative of interaction rate. To do this, Kernel Density Estimation was employed, a technique utilised extensively in geography (Brunsdon 1995). Thus we are assuming that interaction is generated from space and usage, and varies smoothly across our spaces - that locations close to sites of previous interaction carry a preference for future encounters. While we conceive of the encounter rate as a continuous field, in practice we map it onto the same spatial grid produced by our Space Syntax analyses. The distance function used here is a Kernel Density Estimator with a Gaussian function for the kernel - a kernel density estimator works by convolving each data point with a 'kernel' typically a Gaussian - and summing the results. In simpler terms, each point is given a 'fuzzy blob' of interaction probability, and where these blobs overlap, they add up and produce a region of higher interaction rate. Conceptually, this allows us to tie together separate interactions which occur close together, and see them as belonging to the same 'place', or locus, of interaction - and to see more clearly how large or intense that locus is. In producing kernel density estimators, the width of the kernel plays a key role - too small, and we fail to link together points into regions of interaction - too large, and all our regions blur together into one giant smudge of interaction. Our kernel size was $1 \mathrm{~m}$, meaning that two interactions which occurred more than $3 \mathrm{~m}$ apart would not be grouped into the same locus.

\footnotetext{
${ }^{2}$ It should be noted that, while we have used the categorisation of space based on the organisation's use of their buildings, an unsupervised analysis would also be possible, and from this, cluster analyses or unsupervised machine learning methods (such as Self Organising Maps, originally detailed in Kohonen, 1998) could generate those categories organically; this is a subject of ongoing research.
} 
Since both variables (MD and interaction density) are spatial, placing both on the same spatial representation allows for a relational and straightforward interpretation. In order to achieve this layering, a four colour scheme has been selected, as seen in the sample in Figure 1.

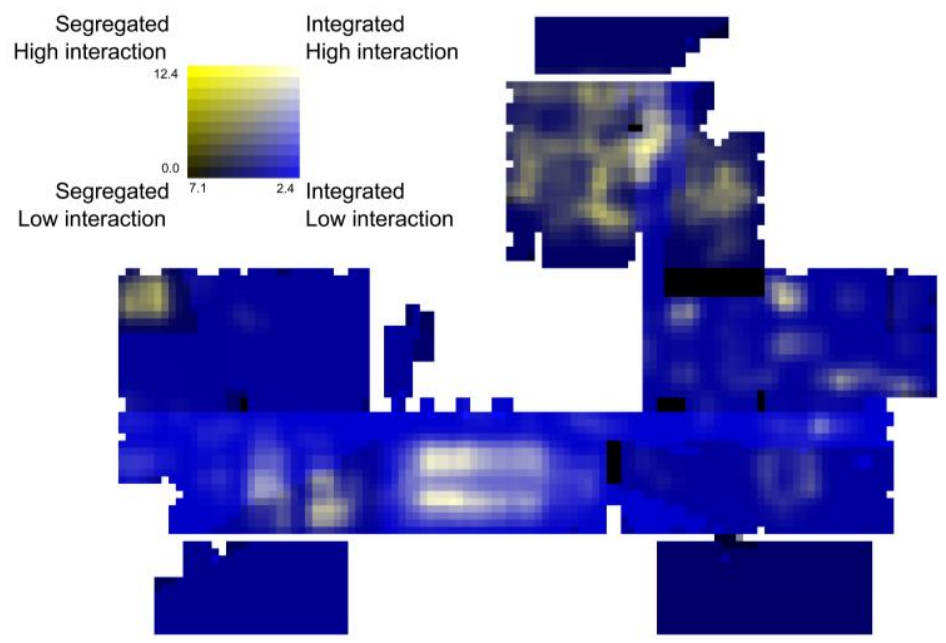

Fig. 1 Sample plan with Visual Mean Depth and interaction density overlay; high interaction in segregated areas is coloured in yellow, turning to white as integration increases; low interaction in segregated areas is coloured in black, turning to blue as integration increases.

Each variable studied is represented by one primary colour, yellow for high levels of interaction and blue for high levels of spatial integration, i.e. low MD and they work additively. When both interaction and integration are low, the pixel will become black; in contrast, when both are high, the colour will turn towards white. The visualisation colour was chosen to be on a colour-blind-safe scale (Brewer et al. 2009).

\section{Interaction Hotspots, Locations and Patterns}

In this section, we will present results from the analysis of interaction patterns. We will start with the big picture of how interactions are distributed in office space across the sample, but will attempt to dig deeper as we go along. Each new arising question will be applied to a more and more trimmed down data set, where we will use types of industry, types of space, floors and different types of interactions as filters to allow us to move from top level patterns to micro-behaviours, organisational cultures and the impact of time. 


\section{The Overall Distribution of Interaction}

Interactions are unevenly distributed throughout the different areas of an office building. We know from previous research that the majority of interactions happen near workstations (Steen et al. 2005, Rashid et al. 2006, Markhede and Koch 2007, Steen 2009) and that attractors (such as coffee machines, photocopiers, canteens, etc.) introduce a positive bias to interactions (Fayard and Weeks 2007).

A similar trend emerges from our data. A total of 161,365 people have been observed in our sample. On average, $30.3 \%$ of people $(48,893)$ present in the office are interacting at any one point in time, which renders interactions an important everyday task at work. As seen in figure $2 \mathrm{a}$, workspaces accumulate the biggest share of those interactions ( $42.6 \%$ for open plan and $12.6 \%$ for cellular), which means that in absolute figures, the highest number of interactions were observed in workspaces (20,751 and 6,056 people interacting respectively). Formal enclosed meeting rooms and shared facilities (such as kitchens, canteens, photocopiers, etc.) account for $23.1 \%$ (11,271 people) and $10.6 \%$ (5,153 people) respectively, while circulation and alternative spaces (such as breakout spaces and informal meeting spaces) attract the lowest number of interactions.

However, it could be argued that workspaces make up the majority of area in an office and as such it would be natural for them to attract most interactions. Therefore we divided the number of interactions occurring in the different areas of the offices in our sample as mentioned above by the overall area provided in each of these categories to understand which of them attracted a disproportionate number of interactions relative to their size; the resulting figures tell us the number of interactions per unit floor area (per $100 \mathrm{~m}^{2}$ ). It could be argued that these spaces are interaction hotspots due to their function. Results are presented in figure $2 b$, providing a comparison of the interaction intensities of different space categories.
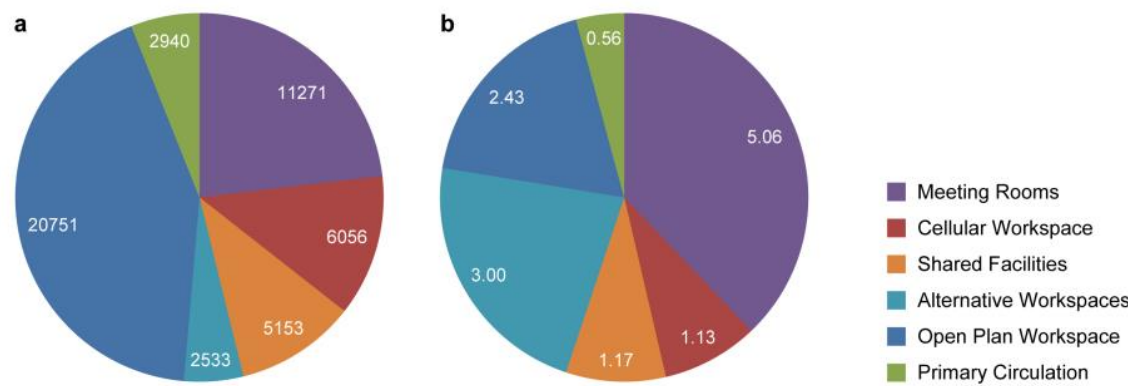

Fig. 2a-b Interaction (split by type of space it occurs in) across all 27 cases in the sample: numbers of people interacting per observation round as a proportion of all people observed (a) and normalised by area provision, i.e. per $100 \mathrm{~m} 2$ (b).

This completely changes the overall picture, since now meeting spaces attract the highest rate of interactions per area $\left(5.06\right.$ people interacting per $100 \mathrm{~m}^{2}$, which equals $38 \%$ of all interactions), followed by alternative meeting spaces ( $22 \%$ or 3.00 
people per $\left.100 \mathrm{~m}^{2}\right)$. Open plan workspaces now only account for $18 \%$ of interactions (2.43 people per $\left.100 \mathrm{~m}^{2}\right)$ with cellular spaces hosting another $8 \%$ (1.13 people per $\left.100 \mathrm{~m}^{2}\right)$. Other facilities and primary circulation make up only one fifth of all interactions. This means that meeting spaces, whether formal or informal, are the places where the highest rate of interaction takes place - clearly an expected result due to the functional programming of these types of areas in the setting of an office. What might come as a surprise is the fact that circulation areas are the least attractive for interactions, which goes against commonly held perceptions. Anecdotally, corridors are often praised as the ideal spot for serendipitous interactions, as the following account from the famous Bell Labs exemplifies: "Traveling the hall's length without encountering a number of acquaintances, problems, diversions and ideas was almost impossible. A physicist on his way to lunch in the cafeteria was like a magnet rolling past iron filings." (Gertner 2012). It might of course be the case that this phenomenon occurs, it is simply not a very predominant pattern overall across our sample.

In addition to the overall spatial distribution of interactions, it is interesting to consider variations across different industries. It could be hypothesized that the distribution, overall occurrence and frequency of interactions varies from one industry to another, depending on their need to exchange ideas, communicate and collaborate. For instance law firms might be expected to have strict rules for handling sensitive data, and might therefore show more proceduralised everyday behaviours. Other companies such as media companies that rely on the quick spreading of information might be expected to tend towards higher levels of interaction due to the need to communicate. Hence we would expect levels of interaction to coincide with the need to coordinate tasks and spread information. We would also expect the locational distribution of interactions to vary by industry.

Despite some relevant variations by industry, the overall distribution of interactions across different functional areas remains comparable to the big picture discussed above for the overall benchmark (see figure 3a-b). Again meeting rooms present themselves as interaction hotspots, however as expected the pattern is not equally pronounced for every industry. Legal firms show the highest preference for formal meeting room interactions, whereas technology companies prefer interactions near their workstations in equal measure. Technology firms also show the highest preference for interacting near facilities such as kitchens, canteens, etc. As expected, the average numbers of people interacting fluctuates by industry (figure $3 b$ ), with companies in the Legal or Media industries showing the most extreme patterns of low levels of interactions (Legal) and very high levels of interaction (Media). This can easily be argued to lie in the nature of the industry and their predominant workflows, as Legal firms tend to deal with sensitive matters, requiring thus a stricter level of privacy and confidentiality, while creative ones, such as those in the Media sector, would encourage interaction as a means to generate new ideas and spread information. 


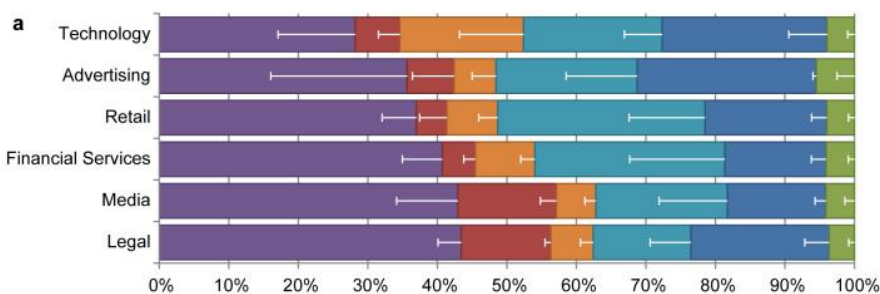

Meeting Rooms Cellular Workspace - Shared Facilities Alternative Workspaces Open Plan Workspace - Primary Circulation

b

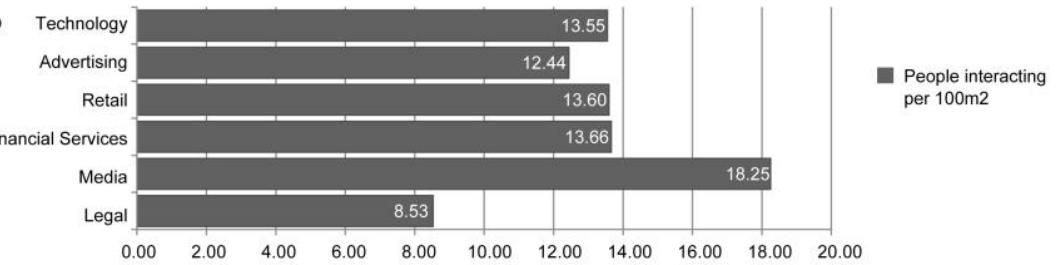

Fig. 3a-b Numbers of people interacting per 100m2, split by types of space and by industry; lines represent standard error (top); average number of people interacting per $100 \mathrm{~m} 2$ per industry (bottom).

\section{The Configurational Logic of Interaction}

But can we pinpoint to specific patterns of interaction that depend on local spatial characteristics? In order to identify such patterns we examine whether interaction systematically relates to visual mean depth. Given that integrated spaces (lower $\mathrm{MD)}$ are more connected and more accessible, we would expect these spaces to engender higher interaction rates due to more opportunities for meeting people, as proposed by previous research studies (Hillier and Grajewski 1990, Toker and Gray 2008). This hypothesis would hold true if pixels that belong to segregated areas also exhibit a narrow and lower range of interaction, while pixels in integrated spaces would tend to cover a larger range and higher densities of interaction. 


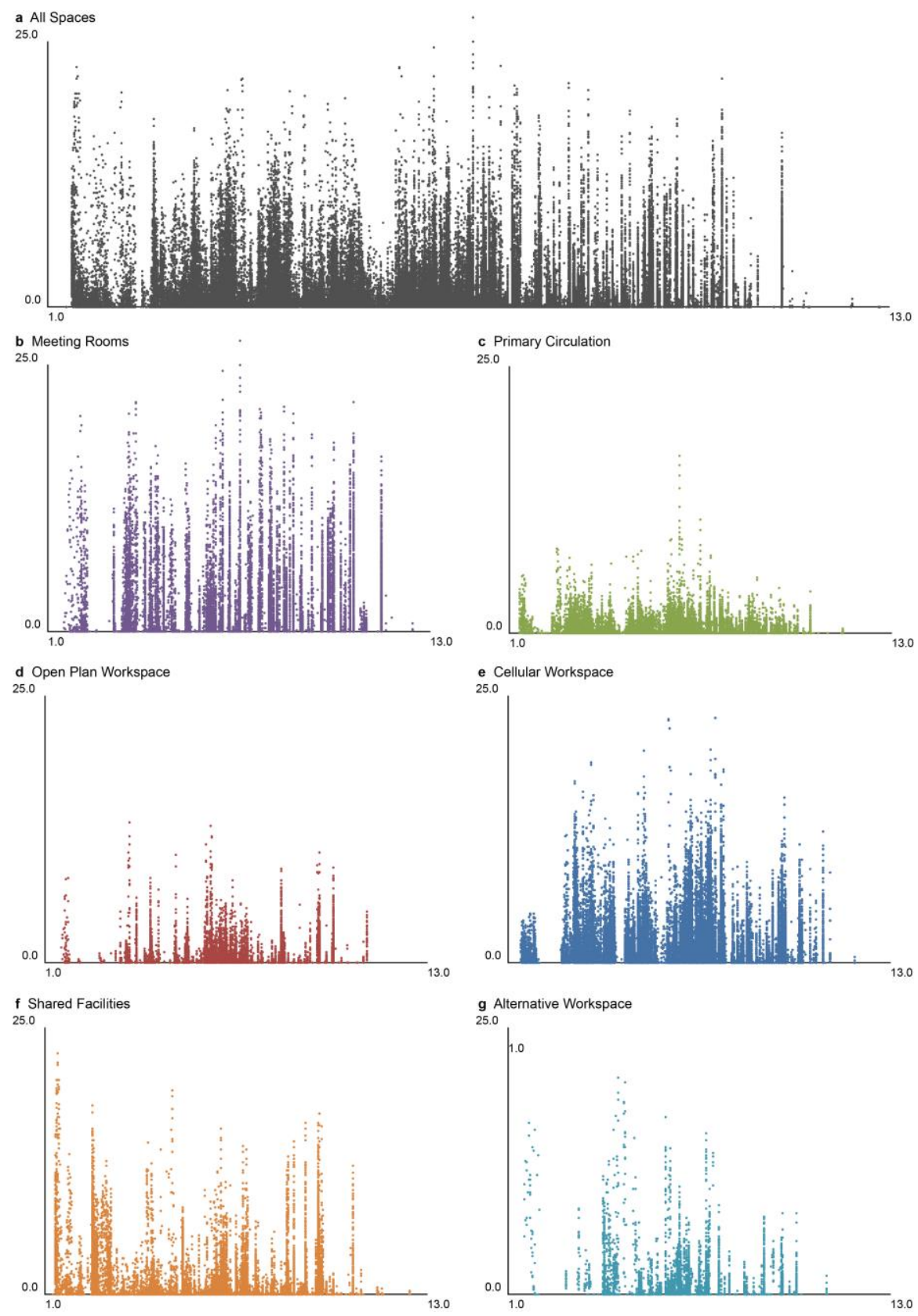

Fig. 4a-g Scatterplots of visual MD (x-axis) and interaction density (y-axis) for all data in the sample (a) (top row), and split by type of space: meeting rooms (b), primary circulation (c), open workspace (d), cellular workspace (e), shared facilities (f) and alternative workspaces (g). 
In figure $4 \mathrm{a}$ it is evident that no clear pattern emerges from plotting MD against interaction density. This is not overly surprising, since configuration certainly is not the only force at play. The influence of attractors is crucial here, because different functional areas attract interactions differently due to their programme. Meeting rooms clearly attract interactions disproportionately, as already shown. The same goes for workspaces: someone's desk is a clear interaction hotspot for that person, since they spend the majority of their time there. In this case we would not expect configuration to matter, since desks are allocated by the organisation. Hence we might expect to see a stronger influence of configuration in certain areas. Therefore the data was analysed separately by the various types of spaces (figures $4 \mathrm{~b}-4 \mathrm{~g}$ ). The fact that the distribution of interaction density appears random in meeting spaces (4b) and workspaces (4d-e) is expected due to their strong functional programme, however we would expect that configuration distributes interactions in primary circulation spaces (4c), shared facilities (4f) and alternative workspaces (4g) according to a spatial logic, i.e. higher interaction densities in integrated areas (low MD) and fewer occurrences of interaction in segregated areas (high MD). However, this is not the case. No pronounced patterns of any sort can be found in the distributions. Further research is clearly needed here.

To delve further into the analysis, in the following section we consider three different projects to see if any clearer patterns appear by examining the data per project and per floor. We studied three buildings, chosen to be approximately comparable in size, each having three floors; this should act to rule out size and configuration effects, which could come with varying numbers of floors. The buildings, however, vary in their overall configuration: example A is a completely open plan office, example B combines open and cellular workspaces, while example $\mathrm{C}$ is mostly cellularised.

For this analysis, we are plotting all interactions taking place in shared facilities on a single floor of each of the three chosen example buildings against their visual mean depth values. This local analysis floor by floor for attractor-based facilities (kitchen, canteen, photocopiers, etc.) reveals a stronger relationship between spatial configuration and interaction. While the range of MD varies due to the spatial configuration of the overall office, within each example the more integrated areas (i.e. of lower mean depth) tend to attract higher interaction densities. The availability of other such facilities in close proximity is also likely to shape interaction density. A large single space as seen in figure 5a attracts higher levels of interaction, in contrast to figure $5 \mathrm{~b}$ where these facilities are fragmented. In figure 5c, where these attractors are few and far apart, interaction is even lower. Still, people across all three randomly chosen examples seem to prefer integrated spaces over segregated spaces as a location for interactions. This means attractors show a synergy effect with integration: people could be argued to frequent those facilities based on their attractiveness and function (such as the need for a cup of tea), however, whether they afford interactions in addition to occupancy seems to be a function of spatial integration. More integrated, larger and more clustered facilities seem to support interactions to a higher degree than segregated, smaller and distributed ones. However as the previous analysis illustrated, this effect can only be seen locally on a floor by floor basis and not within the big dataset as a whole. 

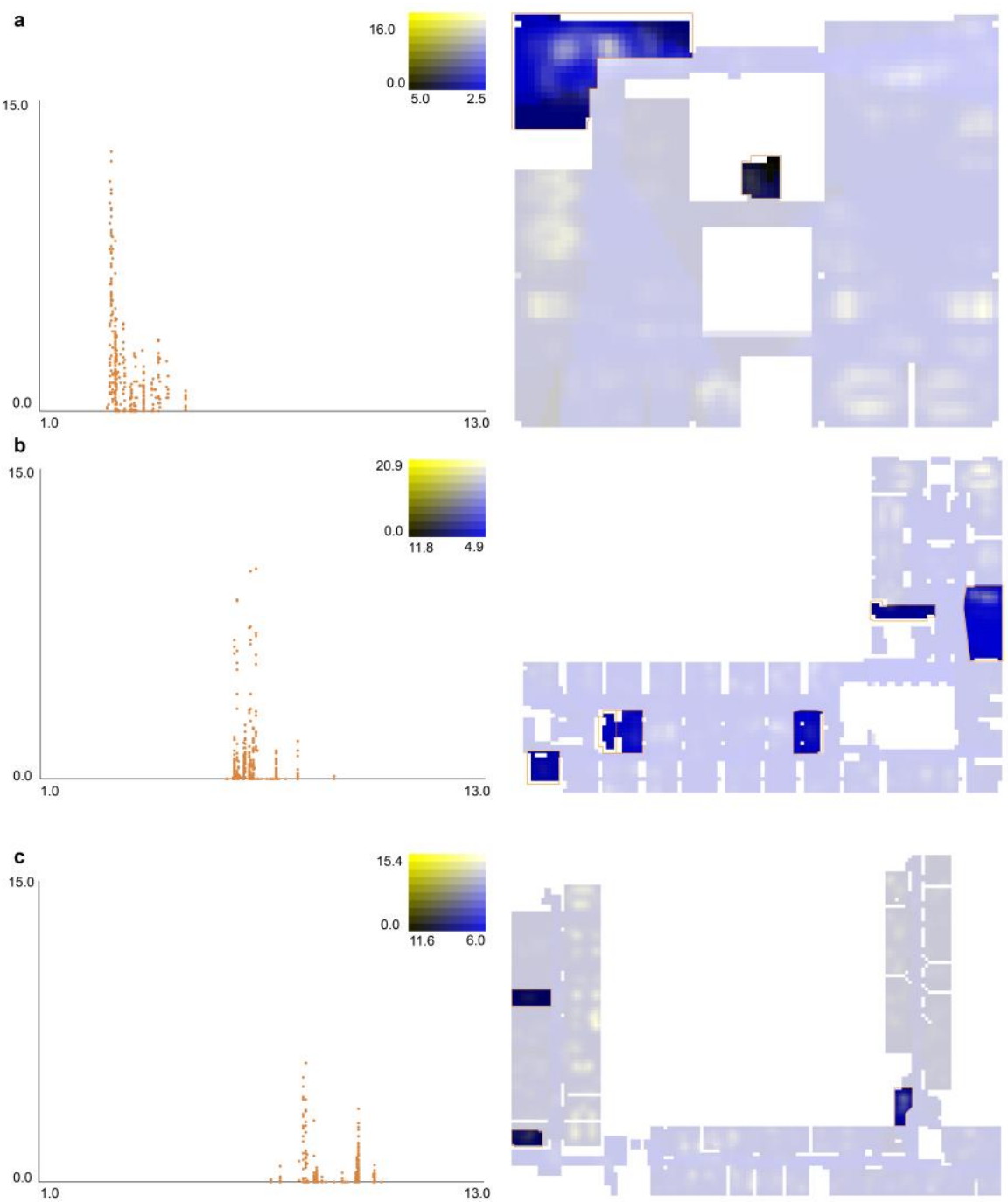

Fig. 5 Left: Visual mean depth (x-axis) and interaction (y-axis) for pixels in shared facilities for three sample floors. Right: Corresponding floor plans with location of facilities highlighted.

\section{The Micro-Behaviours of Interaction}

Since the analysis of localised patterns of interaction seemed fruitful, the next section aims at identifying specific types of interactions in relation to local spatial 
characteristics. Again, it means delving deeper into the data and exploring details more in-depth than in the previous analysis.

In office spaces, the majority of people interacting coincides with the activity of sitting, either in meeting rooms, workspaces, or in other shared facilities such as canteens. As this imposes predefined locations of interaction in the analysis, the dataset is re-examined taking into account only people standing and interacting. Standing may cause fatigue much faster than sitting and is more dynamic and ephemeral in nature, we thus expect that interactions involving people standing are more likely to be brief. They are also likely to depend to a higher degree on the spatial affordances of the environment, since standing could occur anywhere in space.

In this section we therefore want to identify spatial patterns of brief encounters occurring while people are standing. Therefore, we have plotted interaction densities of people standing on a sample floor plan and highlighted emerging categories of interactions in different colours (see figure 6), based on affordances and rationales for interaction as present in the environment. These categories were assigned manually for the purpose of this analysis, with plans to automate this in the future.

The following seven different micro-behavioural interaction patterns have been identified:

1. Recruiting: People standing and interacting in proximity to, or within primary circulation are identified as being engaged in 'recruiting' others into a conversation, a term described by Backhouse and Drew (1992). People walking are in an 'available' state (i.e. not currently focused on work) and are recruited into conversation by their seated colleagues. As this pattern includes people passing-by in a brief conversation, close proximity to the primary circulation perpetuates this behaviour. Interactions like these are considered unplanned, as people passing through may do so at any point in time and will not have planned the interaction in advance. It rather emerges spontaneously based on the opportunity. This pattern is not found in floors which contain cellular workspaces and corridors, since visibility is crucial here.

2. Visiting: An interaction between seated and standing people, away from circulation, described by Penn, Desyllas and Vaughan (1999). An example would be someone visiting a colleague by their desk for a discussion or query. The distance usually kept from circulation allows for longer conversations making these interactions most likely planned.

3. Presenting: Observed in meeting rooms, these are interactions between a person standing and the rest of the (seated) members of the meeting. This behaviour could be an effect of office culture or the type or size of the meeting taking place. It could also signify the type of meeting and the hierarchy and different roles assumed by presenter and audience. 


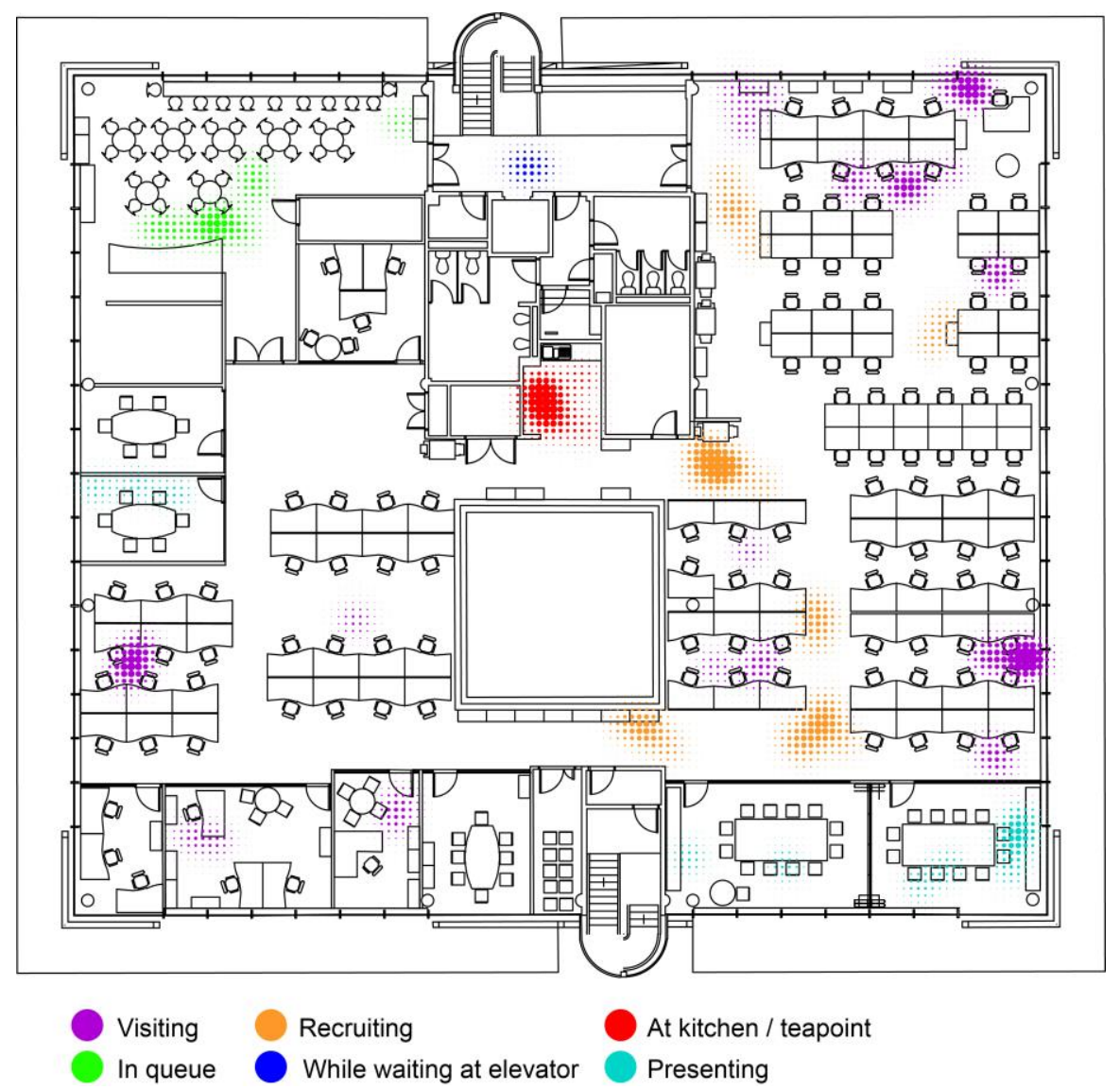

Fig. 6 Hotspots of locations where people stand and interact at the same time

4. Standup meeting: Larger interactions can sometimes be observed taking place in the workspace. They can be brief meetings happening daily in organisations or teams which adopt rapid project development. They may also be quick announcement gatherings, for a team or the whole company. As such, they are considered planned interactions and typically involve larger groups of people.

5. At kitchen / tea point / photocopy room: Attractor based interaction pattern, can be found in any floor that contains a functional kitchen / tea point. Spaces like these are considered shared facilities in this analysis and, as seen later on, have peak interaction levels at lunch time. Photocopy rooms work the same way, but used uniformly through the day. As these attractors are usually part of each floor, the interactions within are very likely to be localised to the floor. These spaces, along with canteens all provide proximity for the people interacting, the necessary privacy and social designation with a purpose to afford informal interactions (Fayard and Weeks, 2007). 
6. In queue: Found in spaces with a food service counter, where a queue is formed. These are usually linear, in the same direction as the counter. Given that canteens with seating area and a service counter are usually one per company (as opposed to one per floor) and in some cases attract people from other companies, the interactions identified here are very likely to be more global in scope.

7. While waiting at elevator: Another 'waiting' interaction in addition to queuing. This is found in front of elevator doors, most likely by people continuing an earlier conversation, or people striking up new ones, just because they are forced to wait at the same location. While the spaces where these interactions happen are not likely to have been designated as 'informal interaction spaces', they tend to be away from workspaces and as in queueing, the common target creates the necessary propinquity.

These patterns shed light on rationales and reasons to interact and allow us to identify the effects spatial affordances can have on interaction, in combination with the presence of others and organisational cultures.

\section{The Time-Space Dependency of Interaction}

A last piece of analysis that can provide insight into how interaction is distributed in office spaces is in its relationship to time. Since we are interested in the dynamics of interactions over the course of a full working week (see figure 7), we only take those datasets into account where observations were conducted for this length of time. These 20 cases (out of 27) cover the full spectrum of industries found in the database, i.e. financial services, technology, advertising, legal, media, retail and public sector.

Clear time-space routines become visible in the analysis (as seen in figure 7a-b), showcasing the daily life of the studied companies and overarching spatial cultures. The overall proportion of people interacting on average (figure 7a) varies slightly throughout the working week with Wednesday being the most interactive day across all cases and industries represented in this sample. Generally, people seem to interact more in the mornings than in the afternoons. Breaking the results down to the type of space they happen in not only reduces the standard error (figure 7a, shown exemplary for open plan workspaces), but also reveals more details about the emerging time-space routines. As shown in figure $7 \mathrm{~b}$, interaction levels start high in workspaces each morning and drop towards noon after which they rise again. Friday evening experiences a particular peak in interactions, possibly due to colleagues chatting about their weekend plans, or finalising last bits and pieces of work before breaking for the weekend. Lunchtime has an inverse effect for the facilities category, as it includes canteens, kitchens and other places where people go for lunch and have lots of chats, creating a clear and recurring peak in the pattern. Interaction in meeting rooms and cellular workspaces rises in mid-morning and mid-afternoon, which is the preferred time for meeting bookings. Interactions in 
primary circulation has varied peaks and troughs, but generally more interactions take place early mornings, at lunch and in the evening, when people are on the move.
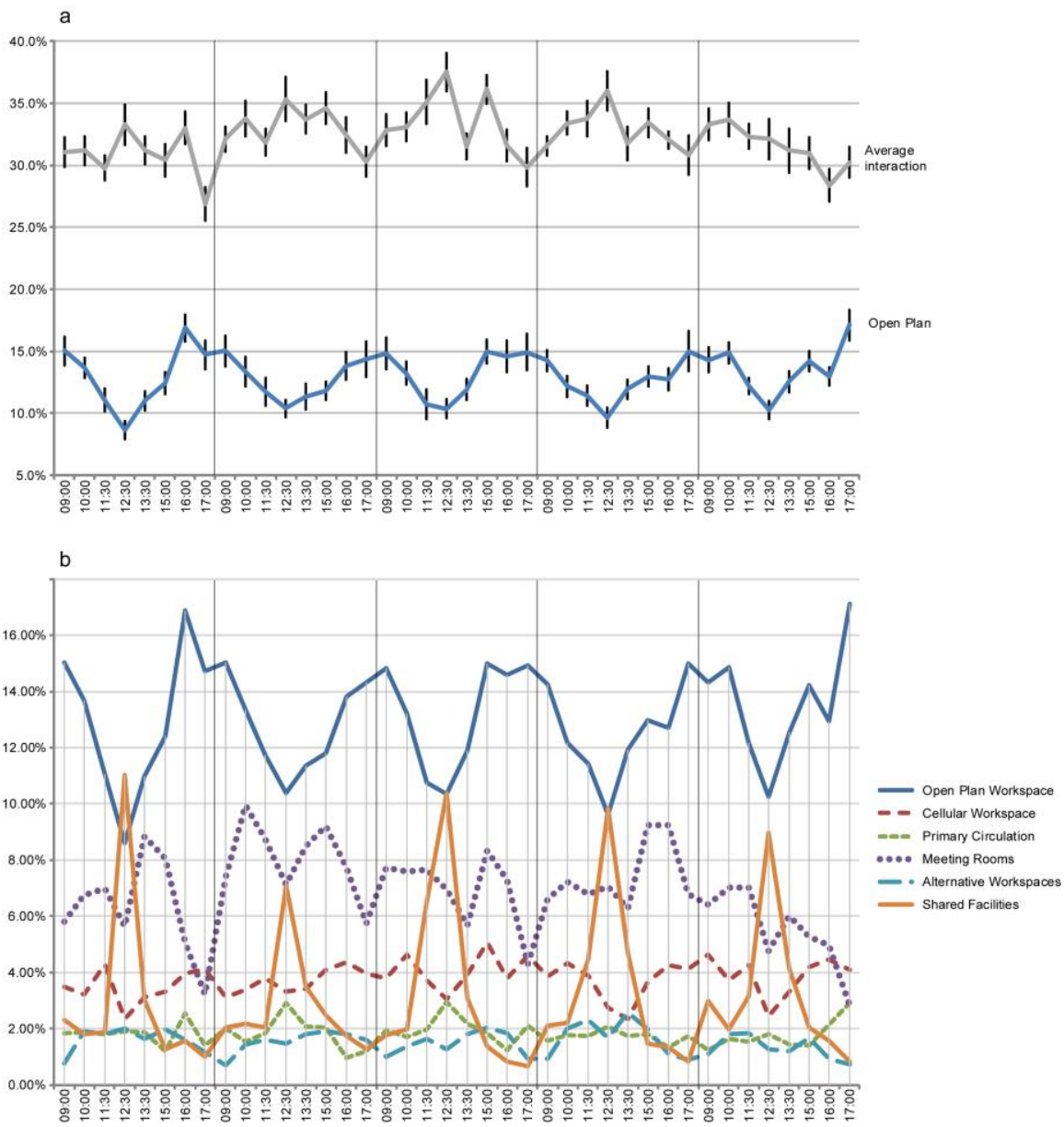

Fig. 7a-b Proportion of people interacting in relation to all people currently in the space, across the week on average and in open-plan spaces; vertical lines represent standard error of the means (top); breakdown per type of space (bottom).

These patterns of behaviour emerge in a top-level analysis of a range of different cases, each with its own way of organising space unique to organisational and sector cultures. Our approach has been to explore the data for underlying, generalizable causative structures, but in some cases our results have run into the limitations of the methodology. 


\section{Overall Patterns and the Problem of Predictability}

In order to facilitate interaction in offices further and in order to support evidencebased design practices - to apply research to practice and use this in new office design - we would need to solve the problem of predictability: how confident can we be about emerging patterns in a new case from looking at the deviations in existing cases?

It is evident from the analysis presented in this book chapter that predictability is not all that easily established. We can expect some relationships to hold in general, but more often than not, trends are not at all clear, especially those at a higher level. We have seen for instance that specific types of spaces will tend to attract more interaction than others (meeting rooms versus workspaces), and narrowing this down to industry or time of day strengthens this relationship. Comparisons to visual mean depth have shown that spatial configuration plays a role in affording interactions, but correlations with this metric were only found on a local floor-by-floor analysis limited to specific spaces (shared facilities).

With regards to this type of analysis, we have merely scratched the surface and more research is required to fully understand the phenomenon of interaction in architectural space. While the sample of cases presented here is still growing and will provide a rich set of opportunities for further exploration, it is debatable whether the right type of information has been captured to get meaningful results. Expanding the dataset in both scope and granularity is one approach (see below); and examining a wider suite of space syntax measures may provide more nuanced insight into the effects of space on functional use.

\section{Future Work and Developments in the Field}

In this final section, we want to provide an outlook on future work in the field; this will be structured to discuss developments in automated behaviour tracking, which is a growing field of interest and could be seen as one form of human computer interaction; to address implications of our findings for the 'sites of HCI'; and to reflect on implications for Space Syntax research.

\section{Automated behaviour tracking}

Automated mechanisms for constant monitoring have the capacity to report in much greater temporal detail than the human observations currently employed. Temporal effects were quite pronounced in the data and could offer a wealth of future research opportunities.

Location and behaviour tracking inside buildings is a dynamic and fast-growing field with emerging and rapidly developing: 
- New technologies (e.g. using magnetic fields such as https://www.indooratlas.com/)

- New measurement systems (e.g. utilising angle-of-arrival signals rather than received signal strength such as http://quuppa.com/ and http://uhopper.com/ or mesh capture such as Xbox Kinect);

- New combinations of sensors (e.g. using heartbeat sensors or electroencephalography (EEG) devices, providing geo-located data related to human emotions responses; see for example, Mavros et al. 2012);

- $\quad$ New form factors (e.g. wristbands)

This could add an understanding of additional layers of information, which might create new insights. In recent years, researchers have begun to synthesise sensor data to build models of institutional dynamics (Pentland 2012). Applying this to space usage could be fruitful, since it is by its nature complex, taking place on multiple social, spatial and temporal levels, requiring a nuanced capture of space, time and interactions with the inclusion of human metrics (physiological and psychophysiological) to fully understand it. An even fuller picture of social interaction would require us to go beyond the activity within the office, linking with people's 'digital footprints' in their life outside work - researchers have begun to use social media and/or mobile telephony data to create detailed views of people's general patterns and preferences (see for instance: Noulas 2015).

The move to automated tracking of usage data, however, will require careful evaluations of what is being measured and to which degree this represents the 'ground truth' of life in the office. Technology is not per se objective, following the observation of Kranzberg (1986): "Technology is neither good nor bad; nor is it neutral." (ibid., p. 545). Instead, it is based on its own assumptions, limitations and built-in capacities, so it could be argued that automated logging again creates a representation that might not provide an 'objective' depiction of reality. Constant monitoring already exists in the 'quantified self' movement, but the use of these technologies by employers to monitor employees will undoubtedly raise ethical concerns in a world already sensitive to issues around inequality, surveillance and personal freedoms. The workplace will need to address these issues rapidly - as indeed will the retail, cultural and entertainment sectors that have already started to employ these tools. By capturing and linking this data, and by managing it in reflective, rigorous and ethical ways, we could harness these powerful new techniques in order to understand human behaviours in offices, both face-to-face but also human computer interaction so that the next generation of office buildings can be adaptive, supportive and enabling human activity and productivity.

\section{Implications for $\mathrm{HCI}$}

Our interaction analysis has demonstrated areas where serendipitous interaction is a natural consequence of the building layout; we have also seen spaces and 
locations which, by their functional assignment, create sociability and interaction opportunities

This knowledge on people's behavioural and spatial preferences in workplace environments can be a useful starting point for new research in the field of HCI. The most obvious bridge from our work to $\mathrm{HCI}$ would be to strategically consider the location of technological interfaces, such as screens or installations in order to maximise their impact. For instance knowing that people hardly interact in circulation spaces might mean to reconsider placing an interface that requires interaction away from corridors and closer to shared facilities. Resources (such as networked tablets with voice interfaces, interactive whiteboards or recording and capture apparatus) could be placed close to informal interaction locations. Another possible application of our research might be in devising systems that automatically detect interactions and provide scenarios for intervention. This tension between automated observation and facilitating agency leads to a spectrum of potential solutions, for instance participants might be directed to available meeting spaces, resources or colleagues available for a chat in a kitchen. Knowing that interactions become less prevalent at certain times of the day or week (e.g. Monday and Friday afternoon) could mean to exploit those times for additional suggestions and interventions. Behavioural monitoring and behaviour change interventions are growing fields of research (for instance: Lathia et al. 2013, Efstratiou et al. 2012).

Bringing architectural research and $\mathrm{HCI}$ together in this context would mean for architects to embrace possibilities of technology and understand how they can enhance social life in the office and for the HCI community to reflect on the affordances of space. Both fields could be united in their endeavours to understand human behaviours to achieve specific outcomes - a well-used space, or a well-used technology.

\section{Implications for Space Syntax}

Furthermore, we can envisage various ways in which our research can push boundaries in the Space Syntax community. Recent contributions have already aimed at methodological innovations that take configurational analysis to the next level by devising methods of visualising and analysing both spatial information and behavioural or organisational aspects in unison. For instance Derix and Jagannath (2014) have combined information of functional allocation (the importance of assets in a bank, for instance) with morphological analysis in a single representation to understand complex relations. Similarly, Kwon and Sailer (2015) have brought behavioural data from where people observe objects inside museum exhibitions and department stores together with a proxemics approach and inter-visibility relations between people. It was shown how different roles emerge (spectators, actors and interactors) and how people in those roles distribute unevenly across space according to spatial configuration. This is highly complementary to the work of Fischer and Hornecker (2012), which classified roles of people in urban encounters in front of media facades (performer, participant, observer) and also worked with a 
framework of proxemics. In addition, new spatial analysis theories close to core interests of $\mathrm{HCI}$ were developed with the intent to unify the understanding of architecture and interaction. Interaction sites, $\mathrm{HCI}$ installations, devices or interpersonal interactions form extra layers of 'augmented information' that both spatial analysts and interaction designers manipulate. In Varoudis (2014) and Varoudis and Penn (2015), the core space syntax analysis used in buildings and small scale neighbourhoods, Visibility Graph Analysis, is extended in order to provide a new methodology able to analyse space and interaction information (in extent, any transpatial or spatiotemporal information) as a single systems where relational asymmetries are explicitly expressed in the analysis (space to space, space to device, space to 'interaction data' in this case).

All of these questions at the interface of behaviours, architectural space and technological interventions yield fascinating opportunities for future research. They raise wider and more complex questions with scope for exploration by $\mathrm{HCI}$ as well as the architectural research community. We hope that the findings reported in this chapter contribute towards these endeavours in providing a baseline of understanding of the complex interplay between space and human-to-human interactions in the workplace.

Acknowledgments This research was partially funded by Innovate UK under the Knowledge Transfer Partnership Scheme (KTP8978 'Big Data in the Office' by UCL and Spacelab, February 2013 - February 2015).

\section{References}

Backhouse, A. and Drew, P. (1992) 'The design implications of social interaction in a workplace setting, Environment and Planning B: Planning and Design, 19, 573-584.

Bernard, H. R. (2000) Social research methods: qualitative and quantitative approaches, Thousand Oaks: SAGE Publications.

Bernard, H. R. and Killworth, P. D. (1993) 'Sampling in Time Allocation Research', Ethnology, 32(2), 207-215.

Bradburn, N. M., Sudman, S., Blair, E. and Stocking, C. (1978) 'Question Threat and Response Bias', Public Opinion Quarterly, 42(2), 221-234.

Brewer, C., and Harrower, M. (2009) "COLORBREWER 2.0." ColorBrewer: Color Advice for Maps. Accessed April 7, 2015. http://www.colorbrewer2.org/.

Brown, C., Efstratiou, C., Leontiadis, I., Quercia, D. and Mascolo, C. (2014a) 'Tracking serendipitous interactions: how individual cultures shape the office', in Proceedings of the 17th ACM conference on Computer supported cooperative work \& social computing, Baltimore, Maryland, USA, 2531641: ACM, 1072-1081.

Brown, C., Efstratiou, C., Leontiadis, I., Quercia, D., Mascolo, C., Scott, J. and Key, P. (2014b) 'The architecture of innovation: Tracking face-to-face interactions with ubicomp technologies', in $A C M$ International Joint Conference on Pervasive and Ubiquitous Computing (Ubicomp 2014), Seattle, Washington, USA, ACM,

Brunsdon, C. (1995) 'Estimating probability surfaces for geographical point data: An adaptive kernel algorithm', Computers \& Geosciences, 21(7), 877-894. 
Cairncross, F. (1997) The Death of Distance: How the Communications Revolution Will Change Our Lives, Cambrige/MA: Harvard University Press.

Curran, K., Furey, E., Lunney, T., Santos, J., Woods, D. and McCaughey, A. (2011) 'An evaluation of indoor location determination technologies', Journal of Location Based Services, 5(2), 61-78.

Derix, C. and Jagannath, P. (2014) 'Digital intuition - Autonomous classifiers for spatial analysis and empirical design', The Journal of Space Syntax, 5(2), 190-215.

Efstratiou, C., Leontiadis, I., Picone, M., Rachuri, K.K., Mascolo, C. and Crowcroft, J. (2012) 'Sense and sensibility in a pervasive world', Pervasive Computing, 406-424.

Fayard, A.-L. and Weeks, J. (2007) 'Photocopiers and Water-coolers. The Affordances of Informal Interaction', Organization Studies, 28(5), 605-634.

Fischer, P. T. and Hornecker, E. (2012) 'Urban HCI: spatial aspects in the design of shared encounters for media facades', in Proceedings of the SIGCHI Conference on Human Factors in Computing Systems, Austin, Texas, USA, 2207719: ACM, 307-316.

Gertner, J. (2012) True Innovation. The New York Times, 25 February 2012

Goffman, E. (1959) The Presentation of Self in Everyday Life, Harmondsworth: Penguin Books.

Grajewski, T. (1992) Space Syntax Observation Manual (2001 unpublished revised edition: L. Vaughan) 2001 unpublished revised edition: L. Vaughan, London: UCL Bartlett and Space Syntax Ltd.

Hall, E. T. (1966) The Hidden Dimension, New York: Doubleday.

Hillier, B. (1996) Space is the machine. A configurational theory of architecture, Cambridge. Online at: http://eprints.ucl.ac.uk/3881/: Cambridge University Press.

Hillier, B. and Grajewski, T. (1990) The application of space syntax to work environments inside buildings: second phase: towards a predictive model, London: Unit for Architectural Studies, The Bartlett School of Architecture and Planning, University College London.

Hillier, B. and Hanson, J. (1984) The social logic of space, Cambridge: Cambridge University Press.

Hillier, B., Hanson, J. and Peponis, J. (1984) 'What do we mean by building function?' in Powell, J. A., Cooper, I. and Lera, S., eds., Designing for building utilisation, London: Spon Ltd, 61-72.

Kohonen, T. (1998) 'The Self-Organizing Map', Neurocomputing, 21 (1-3), 1-6. DOI: 10.1016/S09252312(98)00030-7.

Koutsolampros, P., Sailer, K., Pomeroy, R., Austwick, M., Hudson-Smith, A. and Haslem, R. (2015) 'Spatial Databases: Generating New Insights on Office Design and Human Behaviours in the Workplace', in Karimi, K., Vaughan, L., Sailer, K., Palaiologou, G. and Bolton, T., eds., Proceedings of the 10th International Space Syntax Symposium, London, Space Syntax Laboratory, The Bartlett School of Architecture, University College London, 23:1-23:16.

Kranzberg, M. (1986) 'Technology and History: "Kranzberg's Laws"', Technology and Culture, 27(3), 544-560.

Kwon, S.J. and Sailer, K. (2015) 'Seeing and being seen inside a museum and a department store. A comparison study in visibility and co-presence patterns', in Karimi, K., Vaughan, L., Sailer, K., Palaiologou, G. and Bolton, T., eds., Proceedings of the 10th International Space Syntax Symposium, London, Space Syntax Laboratory, The Bartlett School of Architecture, University College London, 24:1-24:15

Lathia, N., Pejovic, V., Rachuri, K.K., Mascolo, C., Musolesi, M. and Rentfrow, P.J. (2013) 'Smartphones for Large-Scale Behaviour Change Interventions', IEEE Pervasive Computing, 12 (3), 66-73.

Li, N. and Becerik-Gerber, B. (2011) 'Performance-based evaluation of RFID-based indoor location sensing solutions for the built environment', Advanced Engineering Informatics, 25(3), 535-546.

Lopez de Vallejo, I. (2009) Measuring Spatial and Temporal Features of Physical Interaction Dynamics in the Workplace [Doctoral Dissertation], unpublished thesis ( $\mathrm{PhD})$, University College London.

Markhede, H. and Koch, D. (2007) 'Positioning Analysis: Social Structures in Configurative Modelling', in Kubat, A. S., Ertekin, Ö., Güney, Y. I. and Eyüboglu, E., eds., 6th International Space Syntax Symposium, Istanbul, 12-15 June 2007, ITÜ Faculty of Architecture.

Marshall, P., Rogers, Y. and Pantidi, N. (2011) 'Using F-formations to analyse spatial patterns of interaction in physical environments', in Proceedings of the ACM 2011 conference on Computer supported cooperative work, Hangzhou, China, 1958893: ACM, 445-454.

Mavros, P., Coyne, R., Roe, J. and Aspinall, P. (2012) 'Engaging the Brain: Implications of Mobile EEG for Spatial Representation', in Achten, H., Pavlicek, J., Hulin, J. and Matejovska, D., eds., Digital Physicality - Proceedings of the 30th eCAADe Conference, Prague, Czech Republic, Czech Technical University of Prague, Faculty of Architecture, 657-665. 
Namiot, D. (2015) 'On Indoor Positioning', International Journal of Open Information Technologies, 3 (3), 23-26.

Nohria, N. and Eccles, R. G. (1992) 'Face-to-Face: Making Network Organizations Work' in Nohria, N. and Eccles, R. G., eds., Networks and Organizations. Structure, Form, and Action, Boston: Harvard Business School Press, 288-308.

Noulas, A., and Mascolo, C. (2013) 'Exploiting Foursquare and Cellular Data to Infer User Activity in Urban Environments' In: IEEE 14th International Conference on Mobile Data Management (MDM), 1:167-76. DOI: 10.1109/MDM.2013.27.

Olguin, D. O., Waber, B., Taemie, K., Mohan, A., Ara, K. and Pentland, A. (2009) 'Sensible Organizations: Technology and Methodology for Automatically Measuring Organizational Behavior', Systems, Man, and Cybernetics, Part B: Cybernetics, IEEE Transactions on, 39(1), 43 55.

Penn, A., Desyllas, J. \& Vaughan, L. (1999) 'The space of innovation: interaction and communication in the work environment'. Environment and Planning B: Planning and Design, 26, 193-218.

Pentland, A. (2012) 'The New Science of Building Great Teams.' Harvard Business Review. Accessed 13 August 2015. https://hbr.org/2012/04/the-new-science-of-building-great-teams.

Rainie, L. and Wellman, B. (2012) Networked: The New Social Operating System, MIT Press.

Rashid, M., Kampschroer, K., Wineman, J. and Zimring, C. (2006) 'Spatial layout and face-to-face interaction in offices - a study of the mechanisms of spatial effects on face-to-face interaction', Environment and Planning B: Planning and Design, 33, 825-844.

Reiss, A. J. (1971) 'Systematic Observations of Natural Social Phenomena' in Costner, H., ed., Sociological Methodology, San Francisco: Jossey-Bass, 3-33.

Sailer, K. (2007) 'Movement in workplace environments - configurational or programmed?', in Kubat A. S., Ertekin, Ö., Güney, Y. I. and Eyüboglu, E., eds., 6th International Space Syntax Symposium, Istanbul, 12-15 June 2007, ITÜ Faculty of Architecture,

Sailer, K. and McCulloh, I. A. (2012) 'Social Networks and Spatial Configuration - How Office Layouts Drive Social Interaction', Social Networks, 34(1), 47-58.

Sailer, K., Pachilova, R. and Brown, C. (2013) 'Human Versus Machine - Testing Validity and Insights of Manual and Automated Data Gathering Methods in Complex Buildings', in Kim, Y. O., Park, H T. and Seo, K. W., eds., 9th International Space Syntax Symposium, Seoul, Seoul: Sejong University Press,

Sailer, K. and Penn, A. (2009) 'Spatiality and transpatiality in workplace environments', in Koch, D., Marcus, L. and Steen, J., eds., 7th International Space Syntax Symposium, Stockholm, Sweden, Royal Institute of Technology KTH, 095:01 - 095:11.

Sailer, K., Pomeroy, R. and Haslem, R. (2015) 'Ten things you might not know about the workplace: Insights from an evidence-based design practice', Work\&Place(1), 6-9.

Schnädelbach, H. (2012) 'Hybrid spatial topologies', The Journal of Space Syntax, 3(2), 204-222.

Schnädelbach, H., Penn, A., Steadman, P., Benford, S., Koleva, B. and Rodden, T. (2006) 'Moving office: inhabiting a dynamic building', in Proceedings of the 2006 20th anniversary conference on Computer supported cooperative work, Banff, Alberta, Canada, 1180924: ACM, 313-322.

Shklovski, I. and Chang, M. F. (2006) 'Urban Computing--Navigating Space and Context', Computer, 39(9), 36-37.

Steen, J. (2009) 'Spatial and Social Configurations in Offices', in Koch, D., Marcus, L. and Steen, J., eds., 7th International Space Syntax Symposium, Stockholm, Stockholm, TRITA-ARK Forskningspublikation, 107_1 - 107_9.

Steen, J., Blombergsson, M. and Wiklander, J. (2005) 'Useful buildings for office activities', Facilities, 23(3/4), 176-186

Storper, M. and Venables, A. J. (2004) 'Buzz: face-to-face contact and the urban economy', Journal of Economic Geography, 4(4), 351-370.

Toker, U. and Gray, D. O. (2008) 'Innovation spaces: Workspace planning and innovation in U.S. university research centers', Research Policy, 37, 309-329.

Turner, A., Doxa, M., O'Sullivan, D. and Penn, A. (2001) 'From isovists to visibility graphs: a methodology for the analysis of architectural space', Environment and Planning B: Planning and Design, 28(1), 103-121.

Van de Mortel, T. F. (2008) 'Faking it: social desirability response bias in self-report research', Australian Journal of Advanced Nursing, 25(4), 40-48. 
Varoudis, T. (2011) 'Ambient Displays: Influencing Movement Patterns' in Campos, P., Graham, N., Jorge, J., Nunes, N., Palanque, P. and Winckler, M., eds., Human-Computer Interaction - INTERACT 2011Springer Berlin Heidelberg, 52-65.

Varoudis, T. (2012) 'depthmap X: Multi-Platform Spatial Network Analysis Software, OpenSource', 0.30 .

Varoudis, T. (2014) 'Augmented Visibility Graph Analysis - Mixed-directionality graph structure for analysing architectural space.' In: Fusion, Proceedings of the 32nd International Conference on Education and Research in Computer Aided Architectural Design in Europe, 293-302, Vol. 2. eCAADe Conferences, Newcastle upon Tyne, UK: Northumbria University.

Varoudis, T. and Penn, A. (2015) 'Visibility, accessibility and beyond: Next generation visibility graph analysis', in Karimi, K., Vaughan, L., Sailer, K., Palaiologou, G. and Bolton, T., eds., Proceedings of the 10th International Space Syntax Symposium, London, Space Syntax Laboratory, The Bartlett School of Architecture, University College London, 152:1-152:13.

Wu, L., Waber, B., Aral, S., Brynjolfsson, E. and Pentland, A. (2008) 'Mining face-to-face interaction networks using sociometric badges: Predicting productivity in an IT configuration task', SSRN [online], (http://ssrn.com/abstract=1130251)available: doi: [accessed 16 April 2013] 